\title{
Gründung einer Geschäftsstelle für die Deutsche Röntgengesellschaft
}

\author{
Von Prof. Dr. med. Dr. h.c. Paul Gerhardt, Präsident der Deutschen Röntgengesellschaft (DRG) \\ 1988-1991
}

Wenn ich mir die heutigen Strukturen und Zuständigkeiten der Deutschen Röntgengesellschaft (DRG) anschaue, werden bei mir Erinnerungen an die Zeit Ende der 1980er-/Anfang der 1990er-Jahre wach. Auch damals bestand das zentrale Anliegen der DRG darin, den immer rasanteren Entwicklungen in der Medizin und in der Radiologie gerecht zu werden. So hatten wir bereits während des 70. Deutschen Röntgenkongresses 1989 intensiv die Aufgaben der Radiologie und ihre Stellung in der Medizin erörtert und schnell erkannt, dass diese Themen der fortwährenden Aufmerksamkeit und Verhandlungsbereitschaft bedurften.

Umfang und Vielfalt der Tätigkeitsfelder der DRG mögen zwar mit heute nicht vergleichbar sein, aber dennoch hat sich schon damals die Notwendigkeit ergeben, die Arbeit der DRG besser und auch professioneller zu organisieren. Aus diesem Grund hatten die Mitglieder der DRG im Jahr 1989 beschlossen, eine Geschäftsstelle zu gründen, welche daraufhin am 2. Mai 1990 in Neu-Isenburg in der Frankfurter Str. 231 ihre Arbeit aufnahm. Zu den wichtigsten Aufgaben der eigens hierfür eingestellten hauptamtlichen Geschäftsführerin und einer weiteren Mitarbeiterin gehörten die Organisation der Deutschen Röntgenkongresse, der Aufbau von Verbindungen zu den Landesärztekammern, den kassenärztlichen Vereinigungen und der Bundesärztekammer sowie die Verwaltung und Betreuung von seinerzeit 3256 Mitgliedern.

Belastbare Kontakte und gut funktionierende Netzwerke waren bereits damals von nicht unerheblicher Bedeutung, um beispielsweise in den Auseinandersetzungen über die Fachzugehörigkeit der Sonografie und der Interventionsradiologie bestehen zu können. Gleiches galt auch für die Durchführung des Deutschen Röntgenkongresses, der mit Beschluss der DRG-Mitglieder für mindestens 5 Jahre an einem Ort stattfinden und um eine Industrieausstellung erweitert werden sollte.

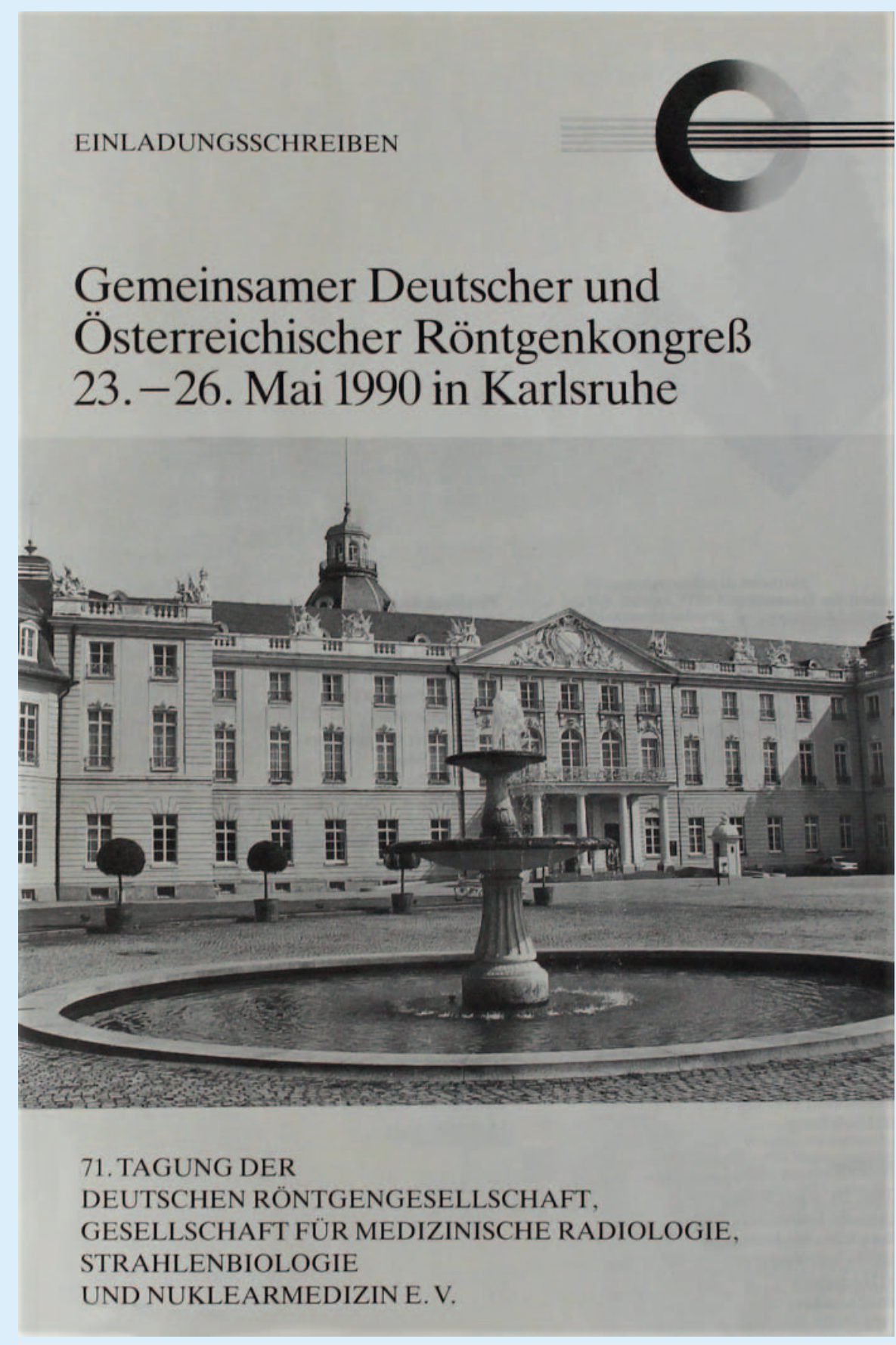

- Abb. 1 Nach einer Pause von 17 Jahren wurde der 71. Deutsche Röntgenkongress erstmals wieder gemeinsam mit der Österreichischen Röntgengesellschaft durchgeführt. ODRG

In diese Zeit fällt auch ein ganz besonderes Ereignis: Im Rahmen des 71. Deutschen Röntgenkongresses 1990 in Karlsruhe nahm die DRG 192 Mitglieder der früheren Gesellschaft für Medizinische Radiologie der DDR auf. Dieser Kongress wurde übrigens auch - nach einer Pause von 17 Jahren - erstmalig wieder mit der Österreichischen Röntgengesellschaft gemeinsam durchgeführt ( $>$ Abb. 1). 
Vieles hat sich verändert, manches ist auch geblieben. Umso wichtiger ist es für die DRG und uns als Radiologen/Radiologinnen, in einen kontinuierlichen Austausch mit dem radiologischen Nachwuchs zu treten. Nicht nur, um Wissen weiterzugeben und das Verständnis untereinander zu befördern, sondern auch, um zu motivieren und gemeinsam den Wandel in der Medizin zu gestalten. Mit dem seit gut 3 Jahren bestehenden „Forum Junge Radiologie“ hat die DRG hierfür eine hervorragende Möglichkeit geschaffen.
In Erinnerung an die zurückliegenden Jahre und die seitdem erfolgreich gestaltete Weiterentwicklung der DRG darf man mehr als zuversichtlich sein, dass die Radiologie auch zukünftig ihren festen Platz im medizinischen Versorgungssystem Deutschlands erhalten, wenn nicht gar ausbauen wird. 